\title{
The Duals of Fusion Frames for Experimental Data Transmission Coding of High Energy Physics
}

\author{
Jinsong Leng, Qixun Guo, and Tingzhu Huang \\ School of Mathematical Sciences, University of Electronic Science and Technology of China, Chengdu 610054, China \\ Correspondence should be addressed to Jinsong Leng; jslengjs@gmail.com
}

Received 9 August 2013; Accepted 27 August 2013

Academic Editor: Carlo Cattani

Copyright (C) 2013 Jinsong Leng et al. This is an open access article distributed under the Creative Commons Attribution License, which permits unrestricted use, distribution, and reproduction in any medium, provided the original work is properly cited.

\begin{abstract}
The experimental data transmission is an important part of high energy physics experiment. In this paper, we connect fusion frames with the experimental data transmission implement of high energy physics. And we research the utilization of fusion frames for data transmission coding which can enhance the transmission efficiency, robust against erasures, and so forth. For this application, we first characterize a class of alternate fusion frames which are duals of a given fusion frame in a Hilbert space. Then, we obtain the matrix representation of the fusion frame operator of a given fusion frame system in a finite-dimensional Hilbert space. By using the matrix representation, we provide an algorithm for constructing the dual fusion frame system with its local dual frames which can be used as data transmission coder in the high energy physics experiments. Finally, we present a simulation example of data coding to show the practicability and validity of our results.
\end{abstract}

\section{Introduction}

Because of the cross-regional feature of high energy physics experiment, there exists a huge amount of data produced in the experiment procedure which needs to be transmitted from each experimental field to the remote center for processing synthetically every day. The relevant technologies in many current data transmission systems are the data transmission protocol GridFTP, the object-related database management system PostgreSQL, the application sever JBoss, and so forth in order to ensure the real-time, reliable, and efficient data transmission [1]. But in many transmission systems, all the signals have to be retransmitted when one or more vectors of data are lost in the transmission process, which leads to wasting a lot of time and resources. Why not use fusion frames? We find that they are natural suitable tools for the experimental data transmission coding of high energy physics. In fact, this application of fusion frames can save more time and resources caused by the retransmission.

Redundancy is an interesting and attractive feature of frames, because it has at least two advantages. First, it makes the construction of various classes of frames flexible; secondly, it can enhance the robustness of encoding data when erasures occur in signal transmissions. So, the theory of frames has been developed rapidly in mathematics and achieved successful applications in various areas of pure and applied sciences and engineering in the past twenty years. We only mention some applications of frames here such as signal and image processing [2], quantization [3], capacity of transmission channel [4-6], coding theory [7-12], and data transmission technology [13].

With the development of signal processing systems, frames are restricted and fusion frames appear. The utility of fusion frames in handling missing data packet erasures problem is shown in [14]. The theory of fusion frames was systematically introduced in $[15,16]$. Since then, many excellent results about the theory and application of fusion frames have been obtained in an amazing speed [15-22]. In fact, fusion frames are generalization of conventional frames and go beyond them, and they have been found to be good tools in large signal processing systems in which distributed or parallel processing is required. For instance, in a coding transmission process, the encoded and quantized data must be put in numbers of packets. When one or more packets are scrumped, lost, or delayed, fusion frames can enhance the robustness to the packet erasures. Furthermore, we can 
see the successful applications of fusion frames in sensors network [23], filter bank [24], transmission coding [14, 25, 26], and so forth. We refer to [27] and the reference therein for more details about the applications of fusion frames.

We first describe how to use fusion frames for transmission coding in experiment of high energy physics to enhance the transmission efficiency and robust against erasures. According to the characteristics of the experimental data transmission system of high energy physics, the distributed, parallel, and fused processing are required in the transmission process. Hence, fusion frames can be applied to coding in the transmission scheme to improve the transmission efficiency, stability, and robust of the whole system.

On the other hand, however, some related problems about fusion frames, especially in applications, are still open. Many excellent results about conventional frames have been achieved and applied successfully, but how to generalize them to fusion frames? It is a tempting subject because of the complexity of the structure of fusion frames compared with conventional frames. For the application of data transmission, we study mainly the dual fusion frames of a given fusion frame and the matrix representations of fusion frame systems in finite-dimensional Hilbert spaces for constructing dual fusion frame systems in this paper.

We outline this paper as follows. In Section 2, we recall the experimental data transmission course of high energy physics and propose a new transmission model in which fusion frame and its dual are used for data coding. Then, we introduce and recall some notations, conceptions, and some basic theory about frames and fusion frame systems. In Section 3, we first introduce a kind of alternate fusion duals based on the definition given in [20]. We investigate and characterize these alternate fusion duals. Then, we consider how to get the matrix representation of the fusion frame operator of a given fusion frame system in a finite-dimensional Hilbert space. So that, based on this matrix representation, a method for construction of the dual fusion frame system with its local dual frames is prescribed. A simulation example is given to show the practicality and validity of these results in experimental data transmission.

\section{Fusion Frames for Experimental Data Transmission Coding of High Energy Physics and Preliminaries}

The main function of fusion frame in data transmission procedure is data coding to implement distributed, parallel, and fused processing of the whole transmission system. A large amount of data produced by experiment sites of high energy physics is encoded by local frames and stored in some packets in the sender sides; the packets from all experiment sites are decoded/processed by dual fusion frame in the center. Based on the conventional transmission system, we establish the structure scheme of the data transmission system by using fusion frames (see Figure 1) and precise the transmission procedure briefly as follows. The original data from the Data Acquisition System is transmitted to the Dropbox for Temporary Directories by the Data Buffer. When the Fetcher finds that there are new data directories in the Dropbox, the original data will be encoded by using a local frame, quantized, and stored into some packets in it. Once the Sending Directories receives these encoded and quantized packets which consist of some vectors from the Dropbox, it will send all these vectors to the processing center and wait the feedback from the receiver. The feedback is sent by the Data Checking Module of the processing center when it confirms that all data from the sender are received. Then it submits all these vectors to the Receiving Directories in which these vectors will be decoded and fusing processed by a fusion frame system and its dual. Finally, all decoded signals are submitted to the Warehouse, and the procedure is over. In the old transmission model, the Data Checking Module of the receiver will check the integrity of these received packets. When it finds that some vectors or coefficients are lost in the transmission process, it will ask the sender retransmit all signals. The re-transmission procedure is unnecessary if a fusion frame and its dual are used for data coding, and a lot of time and resources are saved. Thus, applying fusion frames for data coding in the transmission process can enhance the reliability, efficiency, and robust for erasures. of the transmission system.

Then, let us recall and introduce some basic notations, concepts, and results about frames and fusion frames that are needed for this paper. Let us begin with the concept of frames.

Let $\mathscr{H}$ be a separable (real or complex) Hilbert space. A collection of vectors $F=\left\{f_{i}\right\}_{i \in I} \subset \mathscr{H}$ is called a frame for $\mathscr{H}$ if there exist constants $0<A \leq B<\infty$ such that

$$
A\|f\|^{2} \leq \sum_{i \in I}\left|\left\langle f, f_{i}\right\rangle\right|^{2} \leq B\|f\|^{2} \quad \forall f \in \mathscr{H}
$$

holds for every $f \in \mathscr{H}$, where $I$ denote an index set. The optimal constants (maximal for $A$ and minimal for $B$ ) are called lower and upper frame bounds. If $A=B$, then $F$ is called a tight frame, and it is called a Parseval frame (Sometimes, a Parseval frame is also called a normalized tight frame) when $A=B=1$. A uniform frame is a frame when all the elements in the frame sequence have the same norm.

Given a frame $F=\left\{f_{i}\right\}_{i \in I}$, the operator $\Theta_{F}: \mathscr{H} \rightarrow \ell^{2}(I)$ defined by

$$
\Theta_{F}(f)=\sum_{i \in I}\left\langle f, f_{i}\right\rangle e_{i}
$$

is called the analysis operator of $F$, where $\left\{e_{i}\right\}_{i \in I}$ is the standard orthonormal basis for $\ell^{2}(I)$. The adjoint operator $\Theta_{F}^{*}$ of $\Theta_{F}$ given by

$$
\Theta_{F}^{*}\left(\sum_{i \in I} c_{i} e_{i}\right)=\sum_{i \in I} c_{i} f_{i}
$$

is called the synthesis operator of $F$. If we let $S_{F}=\Theta_{F}^{*} \Theta_{F}$, then we have

$$
S_{F}(f)=\sum_{i \in I}\left\langle f, f_{i}\right\rangle f_{i}, \quad f \in H
$$

Thus, $S_{F}$ is a positive invertible bounded linear operator on $\mathscr{H}$, which is called the frame operator of $F$. 


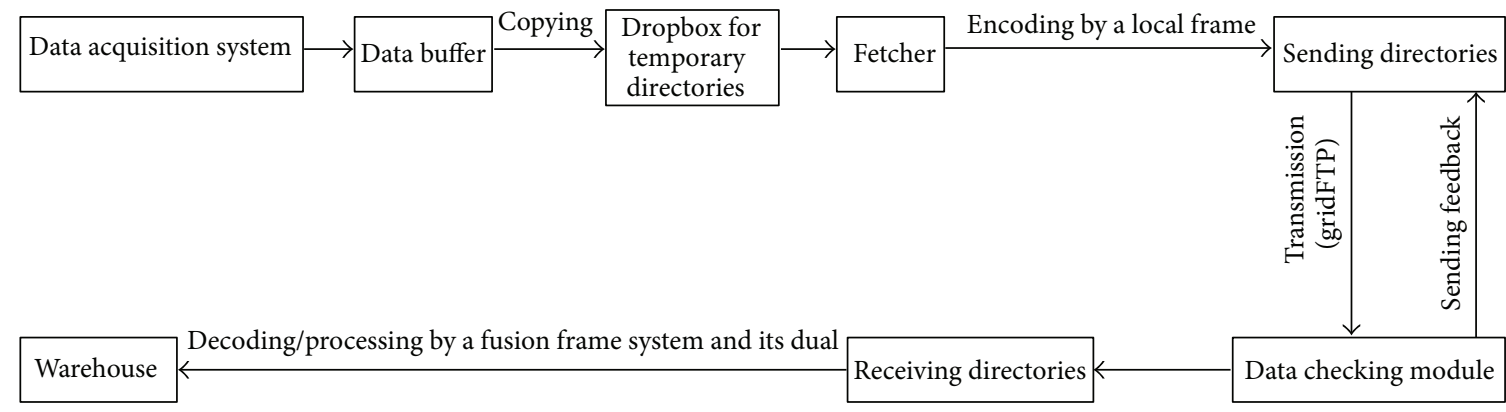

FIgURE 1: The experimental data transmission course of high energy physics.

A collection of vectors $\widetilde{F}=\left\{\widetilde{f}_{i}\right\}_{i \in I}$ in $\mathscr{H}$ is called a dual frame for $F=\left\{f_{i}\right\}_{i \in I}$ if $\widetilde{F}=\left\{\widetilde{f}_{i}\right\}_{i \in I}$ satisfies the reconstruction formula

$$
f=\sum_{i \in I}\left\langle f, f_{i}\right\rangle \tilde{f}_{i}=\sum_{i \in I}\left\langle f, \tilde{f}_{i}\right\rangle f_{i} \quad \forall f \in \mathscr{H} .
$$

A direct calculation yields

$$
f=\sum_{i \in I}\left\langle f, f_{i}\right\rangle S_{F}^{-1}\left(f_{i}\right)=\sum_{i \in I}\left\langle f, S_{F}^{-1}\left(f_{i}\right)\right\rangle f_{i} \quad \forall f \in \mathscr{H} .
$$

This implies that $\left\{S_{F}^{-1}\left(f_{i}\right)\right\}_{i \in I}$ is a dual frame of $\left\{f_{i}\right\}_{i \in I}$. The frame $\left\{S_{F}^{-1}\left(f_{i}\right)\right\}_{i \in I}$ is called the canonical dual frame of $\left\{f_{i}\right\}_{i \in I}$. If a dual frame $\left\{\widetilde{f}_{i}\right\}_{i \in I}$ is not the canonical dual frame, it is also called an alternate dual frame.

A frame $F$ is a tight frame if and only if $S_{F}=\Theta_{F}^{*} \cdot \Theta_{F}=$ $A I_{\mathscr{H}}$ for some positive constant $A$, where $I_{\mathscr{H}}$ is the identity operator. A frame $F$ is a Parseval frame if and only if $S_{F}=$ $\Theta_{F}^{*} \cdot \Theta_{F}=I_{\mathscr{H}}$; that is, the canonical dual of $F$ is itself. So, the analysis operator $\Theta_{F}$ of a Parseval frame is an isometry operator. A linear operator $P$ from a Hilbert space $\mathscr{H}$ to $\mathscr{H}$ is called an orthogonal projection if $P$ is self-adjoint and $P^{2}=P$.

Given a finite frame $F=\left\{f_{i}\right\}_{i=1}^{k}$ in an $n$-dimensional Hilbert space $\mathscr{H}$, then we necessarily have $k \geq n$. When $k=n$, $F$ is automatically a basis of $\mathscr{H}$.

We will use the notation $\mathbb{F}$ when the result being stated holds for both the real number field $\mathbb{R}$ and the complex number field $\mathbb{C}$. When $\mathscr{H}=\mathbb{F}^{n}$, then $f_{i}(i=1,2, \ldots, k)$ are column vectors, and the analysis operator $\Theta_{F}$ for the frame $F=\left\{f_{i}\right\}_{i=1}^{k}$ is a matrix with the row vector $f_{i}^{*}$ as the $i$ th row of the matrix for $i=1,2, \ldots, k$, where the superscript “*” denotes the conjugate-transpose of a vector or a matrix. Relatively, the synthesis operator $\Theta_{F}^{*}$ for the frame $F$ is the conjugate-transpose matrix of $\Theta_{F}$, and the frame operator $S_{F}=\Theta_{F}^{*} \cdot \Theta_{F}$ is an $n \times n$ positive invertible matrix. With respect to a fixed orthonormal basis of $\mathscr{H}$, any element of $\mathscr{H}$ and any linear operator can be expressed by the coordinate vector and the matrix representation. So in most cases we will identify an $n$-dimensional Hilbert space with $\mathbb{F}^{n}$.

Let us now recall the definitions and basic results about fusion frames which are mostly adopted from $[15,16]$.

Definition 1. Let $I$ denote an index set, and let $\left\{W_{i}\right\}_{i \in I}$ be a family of closed subspaces of a Hilbert space $\mathscr{H}$ with a family of weights $\left\{w_{i}\right\}_{i \in I}$ where $w_{i}>0$ for all $i \in I$. Then, $\left\{\left(W_{i}, w_{i}\right)\right\}_{i \in I}$ is called a fusion frame for $\mathscr{H}$ if there exist constants $0<C \leq$ $D<\infty$ such that

$$
C\|f\|^{2} \leq \sum_{i \in I} w_{i}^{2}\left\|P_{W_{i}}(f)\right\|^{2} \leq D\|f\|^{2} \quad \forall f \in \mathscr{H},
$$

where $P_{W_{i}}$ denotes the orthogonal projection onto the $W_{i}$. The constants $C$ and $D$ are called the lower and upper fusion frame bounds. The family $\left\{\left(W_{i}, w_{i}\right)\right\}_{i \in I}$ is called a $C$-tight fusion frame if $C=D$, and it is called a Parseval fusion frame if $C=D=1$. The family $\left\{\left(W_{i}, w_{i}\right)\right\}_{i \in I}$ is called an orthonormal fusion basis if $\mathscr{H}=\oplus_{i \in I} W_{i}$. A Bessel fusion sequence refers to the case when $\left\{\left(W_{i}, w_{i}\right)\right\}_{i \in I}$ has an upper fusion frame bound, but not necessarily a lower bound.

Definition 2. Let $\left\{\left(W_{i}, w_{i}\right)\right\}_{i \in I}$ be a fusion frame for $\mathscr{H}$, and $\left\{f_{i j}\right\}_{j \in J_{i}}$ be a frame of $W_{i}$ where $J_{i}$ are index sets for $i \in I$. Then, $\left\{f_{i j}\right\}_{j \in J_{i}}, i \in I$ are called local frames, and $\left\{\left(W_{i}, w_{i},\left\{f_{i j}\right\}_{j \in J_{i}}\right)\right\}_{i \in I}$ is called a fusion frame system for $\mathscr{H}$. The constants $C$ and $D$ are the associated lower and upper fusion frame bounds if they are the fusion frame bounds for $\left\{\left(W_{i}, w_{i}\right)\right\}_{i \in I}$, and $A$ and $B$ are the local frame bounds if there are the common frame bounds for the local frames $\left\{f_{i j}\right\}_{j \in J_{i}}$ for each $i \in I$. The dual frames $\left\{\widetilde{f}_{i j}\right\}_{j \in J_{i}}, i \in I$ of the local frames in $W_{i}$ are called local dual frames.

The following result shows the relationship between a fusion frame system and its local frames, as well as their frame bounds.

Theorem 3 (c.f. [16], Theorem 2.3). For each $i \in I$, let $W_{i}$ be a closed subspace for $\mathscr{H}$, and let $\left\{f_{i j}\right\}_{j \in J_{i}}$ be a frame for $W_{i}$ with frame bounds $A_{i}$ and $B_{i}$. Suppose that $0<A=$ $\inf _{i \in I} A_{i} \leq \sup _{i \in I} B_{i}=B<\infty$. Then, the following conditions are equivalent.

(i) $\left\{\left(W_{i}, w_{i}\right)\right\}_{i \in I}$ is a fusion frame for $\mathscr{H}$.

(ii) $\left\{w_{i} f_{i j}\right\}_{j \in J_{i}, i \in I}$ is a frame for $\mathscr{H}$.

In particular, if $\left\{\left(W_{i}, w_{i},\left\{f_{i j}\right\}_{j \in J_{i}}\right)\right\}_{i \in I}$ is a fusion frame system for $\mathscr{H}$ with fusion frame bounds $C$ and $D$, then $\left\{w_{i} f_{i j}\right\}_{j \in j_{i}, i \in I}$ is a frame for $\mathscr{H}$ with frame bounds $A C$ and $B D$. If $\left\{w_{i} f_{i j}\right\}_{j \in J_{i}, i \in I}$ is a frame for $\mathscr{H}$ with frame bounds $C$ and $D$, then $\left\{\left(W_{i}, w_{i},\left\{f_{i j}\right\}_{j \in J_{i}}\right)\right\}_{i \in I}$ is a fusion frame system for $\mathscr{H}$ with fusion frame bounds $C / B$ and $D / A$. 
Let $\mathscr{W}=\left\{\left(W_{i}, w_{i}\right)\right\}_{i \in I}$ be a fusion frame for $\mathscr{H}$. The analysis operator $\Theta_{\mathscr{W}}$ is defined by

$$
\Theta_{\mathscr{W}}: \mathscr{H} \longrightarrow\left(\sum_{i \in I} \oplus W_{i}\right)_{\ell_{2}} \text { with } \Theta_{\mathscr{W}}(f)=\left\{w_{i} P_{W_{i}}(f)\right\}_{i \in I}
$$

where

$$
\left(\sum_{i \in I} \oplus W_{i}\right)_{\ell_{2}}=\left\{\left\{f_{i}\right\}_{i \in I} \mid f_{i} \in W_{i},\left\{\left\|f_{i}\right\|\right\}_{i \in I} \in \ell^{2}(I)\right\}
$$

is called the representation space. The synthesis operator $\Theta_{\mathscr{W}}^{*}$ (the adjoint operator of $\Theta_{\mathscr{W}}$ ) can be defined by

$$
\begin{gathered}
\Theta_{\mathscr{W}}^{*}:\left(\sum_{i \in I} \oplus W_{i}\right)_{\ell_{2}} \longrightarrow \mathscr{H} \quad \text { with } \Theta_{\mathscr{W}}^{*}(f)=\sum_{i \in I} w_{i} f_{i}, \\
f=\left\{f_{i}\right\}_{i \in I} \in\left(\sum_{i \in I} \oplus W_{i}\right)_{\ell_{2}} .
\end{gathered}
$$

The fusion frame operator $S_{\mathscr{W}}$ for $\mathscr{W}$ is defined by

$$
S_{\mathscr{W}}(f)=\Theta_{\mathscr{W}}^{*} \Theta_{\mathscr{W}}(f)=\sum_{i \in I} w_{i}^{2} P_{W_{i}}(f)
$$

About dual fusion frames, the following definition was given in [15].

Definition 4. Let $\left\{\left(W_{i}, w_{i}\right)\right\}_{i \in I}$ be a fusion frame for space $\mathscr{H}$ with fusion frame operator $S_{\mathscr{W}}$. Then, $\left\{\left(S_{\mathscr{W}}^{-1} W_{i}, w_{i}\right)\right\}_{i \in I}$ is called the dual fusion frame of $\left\{\left(W_{i}, w_{i}\right)\right\}_{i \in I}$.

The dual fusion frame defined previously satisfies the following reconstruction formula

$$
\begin{aligned}
f & =\sum_{i \in I} w_{i}^{2} S_{\mathscr{W}}^{-1} P_{W_{i}}(f) \\
& =\sum_{i \in I} w_{i}^{2} P_{S_{\mathscr{W}}^{-1} W_{i}} S_{\mathscr{W}}^{-1} P_{W_{i}}(f), \quad f \in \mathscr{H} .
\end{aligned}
$$

Based on (12), the following definition about alternate duals was introduced in [20].

Definition 5. Let $\mathscr{W}=\left\{\left(W_{i}, w_{i}\right)\right\}_{i \in I}$ be a fusion frame for space $\mathscr{H}$ with fusion frame operator $S_{\mathscr{W}}$, and, $\mathscr{V}=\left\{\left(V_{i}, v_{i}\right)\right\}_{i \in I}$ be a Bessel fusion sequence. Then, $\mathscr{V}$ is called an alternate dual of $\mathscr{W}$ if

$$
f=\sum_{i \in I} w_{i} v_{i} P_{V_{i}} S_{\mathscr{W}}^{-1} P_{W_{i}}(f)
$$

holds for all $f \in \mathscr{H}$.

Then, it was proved that $\mathscr{V}$ is also a fusion frame [20]. We will call it an alternate fusion dual of $\mathscr{W}$ in this paper.

\section{A Class of Alternate Fusion Duals and Construction of Dual Fusion Frame Systems}

We first introduce a class of alternate fusion duals $\mathscr{V}=$ $\left\{\left(V_{i}, w_{i}\right)\right\}_{i \in I}$ of a given fusion frame $\mathscr{W}=\left\{\left(W_{i}, w_{i}\right)\right\}_{i \in I}$ which satisfy

$$
P_{V_{i}} S_{\mathscr{W}}^{-1} P_{W_{i}}(f)=P_{S_{\mathscr{W}}^{-1} W_{i}} S_{\mathscr{W}}^{-1} P_{W_{i}}(f), \quad \forall f \in \mathscr{H}, i \in I .
$$

A Bessel fusion sequence which satisfies (14) naturally satisfies (13). So, we can obtain the following obvious result.

Proposition 6. Let $\mathscr{W}=\left\{\left(W_{i}, w_{i}\right)\right\}_{i \in I}$ be a fusion frame for space $\mathscr{H}$ with fusion frame operator $S_{\mathscr{W}}$. If a Bessel fusion sequence $\mathscr{V}=\left\{\left(V_{i}, w_{i}\right)\right\}_{i \in I}$ satisfies (14), then it is an alternate fusion dual of $\mathscr{W}$.

All Bessel fusion sequences satisfying (14) form a special kind of alternate fusion duals of $\mathscr{W}$. The following proposition will characterize these duals.

Proposition 7. Let $\mathscr{W}=\left\{\left(W_{i}, w_{i}\right)\right\}_{i \in I}$ be a fusion frame for space $\mathscr{H}$ with fusion frame operator $S_{\mathscr{W}}$. A Bessel fusion sequence $\mathscr{V}=\left\{\left(V_{i}, w_{i}\right)\right\}_{i \in I}$ satisfies (14) if and only if it has the form

$$
\begin{array}{r}
V_{i}=S_{\mathscr{W}}^{-1} W_{i} \oplus U_{i}, \quad \text { for some subspace } U_{i} \text { of } \mathscr{H} \\
\text { and each } i \in I .
\end{array}
$$

Proof. If (14) holds for any $i \in I$, then we have

$$
P_{V_{i}} S_{\mathscr{W}}^{-1} P_{W_{i}}(f)=P_{S_{\mathscr{W}}^{-1} W_{i}} S_{\mathscr{W}}^{-1} P_{W_{i}}(f)=S_{\mathscr{W}}^{-1} P_{W_{i}}(f), \quad \forall f \in \mathscr{H},
$$

which follows

$$
\left(I_{\mathscr{H}}-P_{V_{i}}\right) S_{\mathscr{W}}^{-1} P_{W_{i}}(f)=P_{V_{i}^{\perp}} S_{\mathscr{W}}^{-1} P_{W_{i}}(f)=0, \quad \forall f \in \mathscr{H} .
$$

For any $f \in S_{\mathscr{W}}^{-1} W_{i}$, there exists $g \in W_{i}$ such that $f=S_{\mathscr{W}}^{-1} g$. Hence,

$$
P_{V_{i}^{\perp}} f=P_{V_{i}^{\perp}} S_{\mathscr{W}}^{-1} g=P_{V_{i}^{\perp}} S_{\mathscr{W}}^{-1} P_{W_{i}} g=0,
$$

which implies that $f \perp V_{i}^{\perp}$. Hence, $S_{\mathscr{W}}^{-1} W_{i} \perp V_{i}^{\perp}$, which follows $S_{\mathscr{W}}^{-1} W_{i} \subset V_{i}$. Let $U_{i}=V_{i}-S_{\mathscr{W}}^{-1} W_{i}$; then for any $f=S_{\mathscr{W}}^{-1} g \in S_{\mathscr{W}}^{-1} W_{i}$, we have

$$
P_{U_{i}} f=\left(P_{V_{i}}-P_{S_{\mathscr{W}}^{-1} W_{i}}\right) S_{\mathscr{W}}^{-1} P_{W_{i}}(g)=0,
$$

which implies

$$
S_{\mathscr{W}}^{-1} W_{i} \perp U_{i}
$$

Hence, $V_{i}=S_{\mathscr{W}}^{-1} W_{i} \oplus U_{i}$.

Conversely, assume that $\mathscr{V}$ satisfies (15). Then for any $f \in$ $\mathscr{H}$ and $i \in I$, since $S_{\mathscr{W}}^{-1} P_{W_{i}}(f) \in S_{\mathscr{W}}^{-1} W_{i}$, we have

$$
P_{V_{i}} S_{\mathscr{W}}^{-1} P_{W_{i}}(f)=P_{S_{\mathscr{W}}^{-1} W_{i}} S_{\mathscr{W}}^{-1} P_{W_{i}}(f),
$$

which implies that (14) holds. 
The following proposition shows that the dual fusion frame $\left\{\left(S_{\mathscr{V}}^{-1} W_{i}, w_{i}\right)\right\}_{i \in I}$ can minimize the projection norm of any $f \in \mathscr{H}$ in the class of alternate fusion duals introduced previously. The property is analogous to Theorem 6.8 of [28] in the case of traditional frames, and its proof is trivial.

Proposition 8. Let $\mathscr{W}=\left\{\left(W_{i}, w_{i}\right)\right\}_{i \in I}$ be a fusion frame for space $\mathscr{H}$ with fusion frame operator $S_{\mathscr{W}}$. Then for any alternate fusion dual $\mathscr{V}=\left\{\left(V_{i}, w_{i}\right)\right\}_{i \in I}$ of $\mathscr{W}$ which satisfies (14), one have

$$
\sum_{i \in I} w_{i}^{2}\left\|P_{S_{\mathscr{W}}^{-1} W_{i}}(f)\right\|^{2} \leq \sum_{i \in I} w_{i}^{2}\left\|P_{V_{i}}(f)\right\|^{2}, \quad \forall f \in \mathscr{H} .
$$

Then, we consider the construction of the dual fusion frame in a finite-dimensional Hilbert space $\mathscr{H}$. In Section 2, we will see that any $n$-dimensional Hilbert space can be identified with $\mathbb{F}^{n}$, and the analysis, synthesis, and frame operator of any conventional frame can be expressed by their matrix representations, respectively. It is essential for this construction to obtain the matrix representation of the fusion frame operator $S_{\mathscr{W}}$ and its inverse which need the local frames. Hence, we will study the construction of the dual fusion frame system $\left\{\left(S_{\mathscr{W}}^{-1} W_{i}, w_{i},\left\{S_{\mathscr{W}}^{-1} f_{i j}\right\}_{j \in J_{i}}\right)\right\}_{i \in I}$ with its local dual frames of a given fusion frame system $\left\{\left(W_{i}, w_{i},\left\{f_{i j}\right\}_{j \in j_{i}}\right\}_{i \in I}\right.$.

Let $\left\{\left(W_{i}, w_{i},\left\{f_{i j}\right\}_{j=1}^{n_{i}}\right)\right\}_{i=1}^{m}$ be a fusion frame system for space $\mathbb{F}^{n}$. Then the analysis operator of the local frame of $W_{i}$ is a $n_{i} \times n$ matrix $\Theta_{F_{i}}$ with $f_{i j}^{*}$ as its $j$ th row, and the $n \times n_{i}$ matrix $\Theta_{F_{i}}^{*}$ is its synthesis operator. Furthermore, the $i$ th local frame operator is an $n \times n$ matrix $S_{F_{i}}=\Theta_{F_{i}}^{*} \Theta_{F_{i}}$.

Remark 9. For the purpose of coding of any $f \in \mathbb{F}^{n}$, any vector of the subspaces of $\mathbb{F}^{n}$ we consider has $n$ elements, and $\Theta_{F_{i}}$ always denotes the analysis operator of the system $\left\{f_{i j}\right\}_{j=1}^{k_{i}}$ in $\mathbb{F}^{n}$ throughout this paper. Hence, it is a $k_{i} \times n$ matrix, not a $k_{i} \times\left(\operatorname{dim} W_{i}\right)$ matrix.

Definition 10. Let $W$ be an $l$-dimensional subspace of $\mathbb{F}^{n}$ with a local frame $F=\left\{f_{i}\right\}_{i=1}^{k}$. $S_{F}$ is the local frame operator of $F$. If there exists an operator $A$ such that $f=S_{F} A(f)=A S_{F}(f)$ holds for all $f \in W$, we call $A$ the inverse of $S_{F}$ in $W$ and denote it by $S_{F}^{-1}$.

For obtaining the local dual frames $\widetilde{f}_{i j}=S_{F_{i}}^{-1}\left(f_{i j}\right)(j=$ $\left.1,2, \ldots, n_{i}, i=1,2, \ldots, m\right)$ of a given fusion frame system $\left\{\left(W_{i}, w_{i},\left\{f_{i j}\right\}_{j \in J_{i}}\right)\right\}_{i \in I}$, where $S_{F_{i}}^{-1}$ is the inverse of $S_{F_{i}}$ in $W_{i}$, we must calculate $S_{F_{i}}^{-1}$ at first. For this purpose, the following lemma holds.

Lemma 11. Let $W$ be an l-dimensional subspace of $\mathbb{F}^{n}$ with an orthonormal basis $\left\{e_{i}\right\}_{i=1}^{l}$ and a frame $F=\left\{f_{i}\right\}_{i=1}^{k}$ with frame bounds $A, B$. Define $L$ to be an $l \times n$ matrix with the vector $e_{i}^{*}$ as its ith row for $i=1,2, \ldots, l$, where $e_{i}^{*}$ is the conjugatetranspose of $e_{i}$. The sequence $G=\left\{g_{i}\right\}_{i=1}^{k}$ is given by $g_{i}=L\left(f_{i}\right)$ for $i=1,2, \ldots, k$. Then, $G$ is a frame of $\mathbb{F}^{l}$ with the same frame bounds as $F$.
Proof. For any $f=\left(a_{1}, a_{2}, \ldots, a_{l}\right)^{*} \in \mathbb{F}^{l}$, we have $L^{*}(f)=$ $\sum_{i=1}^{l} a_{i} e_{i} \in W$, and $\left\|L^{*}(f)\right\|^{2}=\sum_{i=1}^{l}\left|a_{i}\right|^{2}=\|f\|^{2}$, where $L^{*}$ is the conjugate-transpose of $L$. Therefore,

$$
\begin{aligned}
A\|f\|^{2} & =A\left\|L^{*}(f)\right\|^{2} \\
& \leq \sum_{i=1}^{k}\left|\left\langle L^{*}(f), f_{i}\right\rangle\right|^{2}=\sum_{i=1}^{k}\left|\left\langle f, L\left(f_{i}\right)\right\rangle\right|^{2} \\
& =\sum_{i=1}^{k}\left|\left\langle f, g_{i}\right\rangle\right|^{2} \leq B\left\|L^{*}(f)\right\|^{2}=B\|f\|^{2},
\end{aligned}
$$

as required.

Theorem 12. Let $W$ be an l-dimensional subspace of $\mathbb{F}^{n}$ with an orthonormal basis $\left\{e_{i}\right\}_{i=1}^{l}$ and a frame $F=\left\{f_{i}\right\}_{i=1}^{k}$. L is defined as the previously mentiond lemma. $S_{F}$ is the frame operator of $F$. Then,

$$
S_{F}^{-1}=L^{*}\left(L S_{F} L^{*}\right)^{-1} L,
$$

is the inverse of $S_{F}$ in $W$. Moreover, the orthogonal projection $P_{W}$ from $F^{n}$ onto $W$ is $P_{W}=S_{F}^{-1} S_{F}=S_{F} S_{F}^{-1}=L^{*} L$.

Proof. Let $\Theta_{F}$ and $\Theta_{F}^{*}$ be the analysis operator and synthesis operator of $F$, respectively; then $L \Theta_{F}^{*}=\left(L\left(f_{1}\right), L\left(f_{2}\right), \ldots\right.$, $\left.L\left(f_{k}\right)\right)$ is the synthesis operator of $G=\left\{g_{i}=L\left(f_{i}\right)\right\}_{i=1}^{k}$ which is denoted by $\Theta_{G}^{*}$. By the previous lemma lemma, $G$ is the frame of $\mathbb{F}^{l}$; hence, the matrix $S_{G}=\Theta_{G}^{*} \Theta_{G}=L \Theta_{F}^{*} \Theta_{F} L^{*}=$ $L S_{F} L^{*}$ which is the frame operator of $G$ is invertible. Denote $L^{*}\left(L S_{F} L^{*}\right)^{-1} L$ by $S_{F}^{-1}$.

For any $f \in W$, we have

$$
f=\sum_{i=1}^{l}\left\langle f, e_{i}\right\rangle e_{i}=L^{*} L(f) .
$$

Therefore, we can get

$$
\begin{aligned}
& S_{F}^{-1} S_{F}(f) \\
& =L^{*}\left(L S_{F} L^{*}\right)^{-1} L S_{F}(f) \\
& =L^{*}\left(L S_{F} L^{*}\right)^{-1} L S_{F} L^{*} L(f)=L^{*} L(f)=f, \\
& S_{F} S_{F}^{-1}(f) \\
& \quad=S_{F} L^{*}\left(L S_{F} L^{*}\right)^{-1} L(f) \\
& =L^{*} L S_{F} L^{*}\left(L S_{F} L^{*}\right)^{-1} L(f)=L^{*} L(f)=f ;
\end{aligned}
$$

hence, $S_{F}^{-1}$ is the inverse of $S_{F}$ in $W$.

Moreover, for any $f \in \mathbb{F}^{n}$, its orthogonal projection onto $W$ is

$$
\begin{aligned}
P_{W}(f) & =\sum_{i=1}^{k}\left\langle P_{W}(f), f_{i}\right\rangle S_{F}^{-1}\left(f_{i}\right) \\
& =\sum_{i=1}^{k}\left\langle f, f_{i}\right\rangle S_{F}^{-1}\left(f_{i}\right)=S_{F}^{-1} \Theta_{F}^{*} \Theta_{F}(f)=S_{F}^{-1} S_{F}(f)
\end{aligned}
$$




$$
\begin{aligned}
& =\sum_{i=1}^{k}\left\langle f, S_{F}^{-1}\left(f_{i}\right)\right\rangle f_{i}=\Theta_{F}^{*} \Theta_{F} S_{F}^{-1}(f) \\
& =S_{F} S_{F}^{-1}(f)=\sum_{i=1}^{l}\left\langle f, e_{i}\right\rangle e_{i}=L^{*} L(f),
\end{aligned}
$$

as claimed.

The proof of the following proposition is straightforward, by using Proposition 2.6 of [16] and the previous theorem, we omit it.

Proposition 13. Let $\left\{\left(W_{i}, w_{i}, F_{i}=\left\{f_{i j}\right\}_{j=1}^{n_{i}}\right)\right\}_{i=1}^{m}$ be a fusion frame system for $\mathbb{F}^{n}$, and let $\widetilde{F}_{i}=\left\{\widetilde{f}_{i j}\right\}_{j \in J_{i}}, i \in I$ be the local dual frames given by $\tilde{f}_{i j}=S_{F_{i}}^{-1}\left(f_{i j}\right)$ for all $j=1,2, \ldots, n_{i}$, $i=1,2, \ldots, m$. Then, the matrix representation of the fusion frame operator is given by

$$
\begin{aligned}
S_{\mathscr{W}} & =\sum_{i \in I} w_{i}^{2} \Theta_{\widetilde{F}_{i}}^{*} \Theta_{F_{i}}=\sum_{i \in I} w_{i}^{2} \Theta_{F_{i}}^{*} \Theta_{\widetilde{F}_{i}} \\
& =\sum_{i \in I} w_{i}^{2} S_{F_{i}}^{-1} S_{F_{i}}=\sum_{i \in I} w_{i}^{2} S_{F_{i}} S_{F_{i}}^{-1},
\end{aligned}
$$

where $\Theta_{F_{i}}$ and $\Theta_{\widetilde{F}_{i}}$ are the analysis operators of $F_{i}$ and $\widetilde{F}_{i}$, respectively, and $S_{F_{i}}$ is the frame operator of $F_{i}$ for each $i \in I$.

Given a fusion frame system $\left\{\left(W_{i}, w_{i}, F_{i}=\left\{f_{i j}\right\}_{j=1}^{n_{i}}\right)\right\}_{i=1}^{m}$ of a finite-dimensional Hilbert space $\mathbb{F}^{n}$, we summarize the previous results to provide the concrete algorithm to construct its dual fusion frame system $\left\{\left(S_{\mathscr{W}}^{-1} W_{i}, w_{i}, S_{\mathscr{W}}^{-1} F_{i}=\right.\right.$ $\left.\left.\left\{S_{\mathscr{W}}^{-1} f_{i j}\right\}_{j=1}^{n_{i}}\right)\right\}_{i=1}^{m}$ with its local dual frames as follows.

Step 1. For each $i=1,2, \ldots, m$, search the maximally linear independent subset of $F_{i}$; we denote it by $G_{i}=\left\{g_{i j}\right\}_{j=1}^{l_{i}}$.

Step 2. Use the Gram-Schmidt process on $G_{i}$ to compute an orthonormal basis for $W_{i}$; we denote it by $R_{i}=\left\{e_{i j}\right\}_{j=1}^{l_{i}}$. Construct the matrix $L_{i}$ constituted by this basis as follows:

$$
L_{i}=\left[\begin{array}{c}
\longleftarrow e_{i 1}^{*} \longrightarrow \\
e_{i 2}^{*} \longrightarrow \\
\vdots \\
\longleftarrow e_{i l_{i}}^{*} \longrightarrow
\end{array}\right]
$$

Step 3. Since $P_{W_{i}}=L_{i}^{*} L_{i}$ by Theorem 12, we have $S_{\mathscr{W}}=$ $\sum_{i=1}^{m} w_{i}^{2} L_{i}^{*} L_{i}$.

Step 4. Compute the local frames $S_{\mathscr{W}}^{-1} F_{i}=\left\{S_{\mathscr{W}}^{-1}\left(f_{i j}\right)\right\}_{j=1}^{n_{i}}$ of $\left\{\left(S_{\mathscr{W}}^{-1} W_{i}, w_{i}\right)\right\}_{i=1}^{m}$ for $i=1,2, \ldots, m$.

Step 5. Use formula (24) to calculate the inverse of all local frame operators $S_{S_{\mathscr{W}^{-1} F_{i}}^{-1}}$ in $S_{\mathscr{W}^{-1}} W_{i}$ for $i=1,2, \ldots, m$.
Step 6. Calculate the local dual frames $S_{S_{\mathscr{W}^{-1} F_{i}}}^{-1}\left(S_{\mathscr{W}}^{-1} F_{i}\right)=$ $\left\{S_{S_{\mathscr{W}}^{-1} F_{i}}^{-1}\left(S_{\mathscr{W}}^{-1}\left(f_{i j}\right)\right)\right\}_{j=1}^{n_{i}}$, of $\left\{\left(S_{\mathscr{W}}^{-1} W_{i}, w_{i}\right)\right\}_{i=1}^{m}$ for $i=1,2, \ldots, m$, as required.

The fusion frame system of the following example is given in [21].

Example 14. Assume that $n=4, m=2$, and $n_{1}=n_{2}=3$. The fusion frame system $\left\{\left(W_{i}, w_{i}, F_{i}=\left\{f_{i j}\right\}_{j=1}^{3}\right)\right\}_{i=1}^{2}$ is given by $w_{1}=w_{2}=1$ and

$$
\begin{gathered}
f_{11}=\left(\frac{\sqrt{6}}{6},-\frac{\sqrt{6}}{6}, \frac{\sqrt{6}}{6},-\frac{\sqrt{3}}{3}\right)^{T}, \\
f_{12}=\left(\frac{\sqrt{6}}{6},-\frac{\sqrt{6}}{6},-\frac{\sqrt{6}}{6}, \frac{\sqrt{3}}{3}\right)^{T}, \\
f_{13}=\left(\frac{\sqrt{6}}{6},-\frac{\sqrt{6}}{6}, 0,0\right)^{T}, \quad f_{21}=\left(\frac{6}{6}, \frac{6}{6}, 0,0\right)^{T}, \\
f_{22}=\left(0,0,-\frac{\sqrt{6}}{3},-\frac{\sqrt{3}}{3}\right)^{T}, \quad f_{23}=\left(\frac{\sqrt{3}}{3}, \frac{\sqrt{3}}{3}, 0,0\right)^{T} .
\end{gathered}
$$

The maximally linear independent subsets of $F_{1}$ and $F_{2}$ are $\left\{f_{11}, f_{12}\right\}$ and $\left\{f_{21}, f_{22}\right\}$, respectively. Applying the GramSchmidt process on them, we get the orthonormal bases of $W_{1}$ and $W_{2}$ as follows:

$$
\begin{gathered}
e_{11}=\left(\sqrt{\frac{1}{5}},-\sqrt{\frac{1}{5}}, \sqrt{\frac{1}{5}},-\sqrt{\frac{2}{5}}\right)^{T}, \\
e_{12}=\left(\sqrt{\frac{3}{10}},-\sqrt{\frac{3}{10}},-\sqrt{\frac{2}{15}}, \sqrt{\frac{4}{15}}\right)^{T}, \\
e_{21}=\left(\frac{1}{\sqrt{2}}, \frac{1}{\sqrt{2}}, 0,0\right)^{T}, \quad e_{22}=\left(0,0,-\frac{\sqrt{6}}{3},-\frac{\sqrt{3}}{3}\right)^{T} .
\end{gathered}
$$

So that

$$
\begin{gathered}
L_{1}=\left[\begin{array}{cccc}
\sqrt{\frac{1}{5}} & -\sqrt{\frac{1}{5}} & \sqrt{\frac{1}{5}} & -\sqrt{\frac{2}{5}} \\
\sqrt{\frac{3}{10}} & -\sqrt{\frac{3}{10}} & -\sqrt{\frac{2}{15}} & \sqrt{\frac{4}{15}}
\end{array}\right], \\
L_{2}=\left[\begin{array}{cccc}
\frac{1}{\sqrt{2}} & \frac{1}{\sqrt{2}} & 0 & 0 \\
0 & 0 & -\frac{\sqrt{6}}{3} & -\frac{\sqrt{3}}{3}
\end{array}\right] .
\end{gathered}
$$

Then, we have $S_{\mathscr{W}}=\sum_{i=1}^{2} w_{i}^{2} L_{i}^{*} L_{i}=I$, where $I$ is the identity matrix, which implies that the fusion frame system $\left\{\left(W_{i}, w_{i},\left\{f_{i j}\right\}_{j=1}^{3}\right)\right\}_{i=1}^{2}$ is a Parseval fusion frame, and the local frames of the dual fusion frame system $\left\{\left(S_{\mathscr{W}}^{-1} W_{i}, w_{i},\left\{S_{\mathscr{W}}^{-1} f_{i j}\right\}_{j=1}^{3}\right)\right\}_{i=1}^{2}$ are $S_{\mathscr{W}}^{-1} F_{i}=F_{i}$ for $i=1,2$. 
Since

we can get

$$
S_{F_{1}}=\Theta_{F_{1}}^{*} \Theta_{F_{1}}=\left[\begin{array}{cccc}
\frac{1}{2} & -\frac{1}{2} & 0 & 0 \\
-\frac{1}{2} & \frac{1}{2} & 0 & 0 \\
0 & 0 & \frac{1}{3} & -\frac{\sqrt{2}}{3} \\
0 & 0 & -\frac{\sqrt{2}}{3} & \frac{2}{3}
\end{array}\right] \text {, }
$$

$$
\begin{aligned}
& \Theta_{F_{2}}=\left[\begin{array}{cccc}
\frac{6}{6} & \frac{6}{6} & 0 & 0 \\
0 & 0 & -\frac{\sqrt{6}}{3} & -\frac{\sqrt{3}}{3} \\
\frac{\sqrt{3}}{3} & \frac{\sqrt{3}}{3} & 0 & 0
\end{array}\right] \text {, } \\
& \Theta_{F_{1}}^{*}=\left[\begin{array}{ccc}
\frac{\sqrt{6}}{6} & \frac{\sqrt{6}}{6} & \frac{\sqrt{6}}{6} \\
-\frac{\sqrt{6}}{6} & -\frac{\sqrt{6}}{6} & -\frac{\sqrt{6}}{6} \\
\frac{\sqrt{6}}{6} & -\frac{\sqrt{6}}{6} & 0 \\
-\frac{\sqrt{3}}{3} & \frac{\sqrt{3}}{3} & 0
\end{array}\right], \\
& \Theta_{F_{2}}^{*}=\left[\begin{array}{ccc}
\frac{6}{6} & 0 & \frac{\sqrt{3}}{3} \\
\frac{6}{6} & 0 & \frac{\sqrt{3}}{3} \\
0 & -\frac{\sqrt{6}}{3} & 0 \\
0 & -\frac{\sqrt{3}}{3} & 0
\end{array}\right],
\end{aligned}
$$

$$
S_{F_{2}}=\Theta_{F_{2}}^{*} \Theta_{F_{2}}=\left[\begin{array}{cccc}
\frac{4}{3} & \frac{4}{3} & 0 & 0 \\
\frac{4}{3} & \frac{4}{3} & 0 & 0 \\
0 & 0 & \frac{2}{3} & \frac{\sqrt{2}}{3} \\
0 & 0 & \frac{\sqrt{2}}{3} & \frac{1}{3}
\end{array}\right] .
$$

By using (24), we obtain

$$
\begin{aligned}
S_{F_{1}}^{-1}=L_{1}^{*}\left(L_{1} S_{F_{1}} L_{1}^{*}\right)^{-1} L_{1}= & {\left[\begin{array}{cccc}
\frac{1}{2} & -\frac{1}{2} & 0 & 0 \\
-\frac{1}{2} & \frac{1}{2} & 0 & 0 \\
0 & 0 & \frac{1}{3} & -\frac{\sqrt{2}}{3} \\
0 & 0 & -\frac{\sqrt{2}}{3} & \frac{2}{3}
\end{array}\right], } \\
S_{F_{2}}^{-1}=L_{2}^{*}\left(L_{2} S_{F_{2}} L_{2}^{*}\right)^{-1} L_{2} & =\left[\begin{array}{cccc}
\frac{3}{16} & \frac{3}{16} & 0 & 0 \\
\frac{3}{16} & \frac{3}{16} & 0 & 0 \\
0 & 0 & \frac{2}{3} & \frac{\sqrt{2}}{3} \\
0 & 0 & \frac{\sqrt{2}}{3} & \frac{1}{3}
\end{array}\right] .
\end{aligned}
$$

Hence, the local dual frame of $F_{1}$ is

$$
\begin{gathered}
\tilde{f}_{11}=S_{F_{1}}^{-1} f_{11}=f_{11}, \quad \tilde{f}_{12}=S_{F_{1}}^{-1} f_{12}=f_{12}, \\
\tilde{f}_{13}=S_{F_{1}}^{-1} f_{13}=f_{13},
\end{gathered}
$$

the local dual frame of $F_{2}$ is

$$
\begin{gathered}
\tilde{f}_{21}=S_{F_{2}}^{-1} f_{21}=\left(\frac{3}{8}, \frac{3}{8}, 0,0\right)^{T}, \quad \tilde{f}_{22}=S_{F_{2}}^{-1} f_{22}=f_{22}, \\
\tilde{f}_{23}=S_{F_{2}}^{-1} f_{23}=\left(\frac{\sqrt{3}}{8}, \frac{\sqrt{3}}{8}, 0,0\right)^{T} .
\end{gathered}
$$

They are also the local dual frames of the dual fusion frame system $\left\{\left(S_{\mathscr{W}}^{-1} W_{i}, w_{i},\left\{S_{\mathscr{W}}^{-1} f_{i j}\right\}_{j=1}^{3}\right)\right\}_{i=1}^{2}$.

The quark-gluon plasma is a state of the extremely dense matter which contains the quarks and gluons in high energy physics. The gray image of quark-gluon plasma is shown in Figure 2. We encode the data of the image by using the local frames of the fusion frame given by this example. Suppose that the fist element of every local vector is lost in the transmission process. Then, we decode the received data by using the dual fusion frame computed by this example. The reconstructed image is shown in Figure 3. One can observe the reconstruction effect by comparing the two figures. 


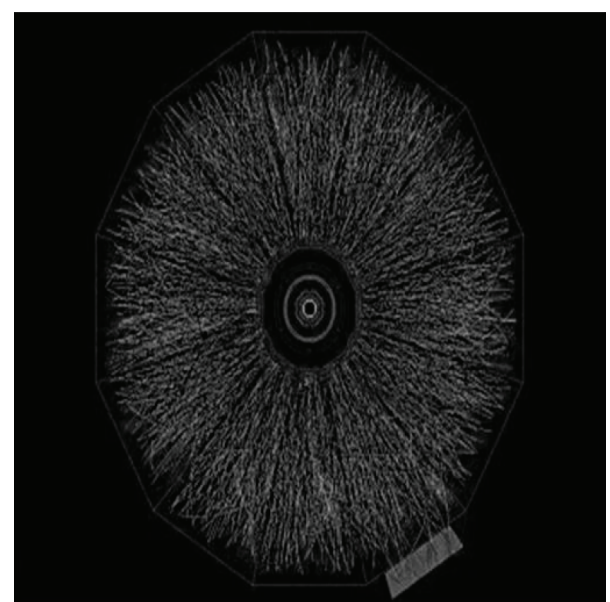

FIGURE 2: The original gray image of quark-gluon plasma.

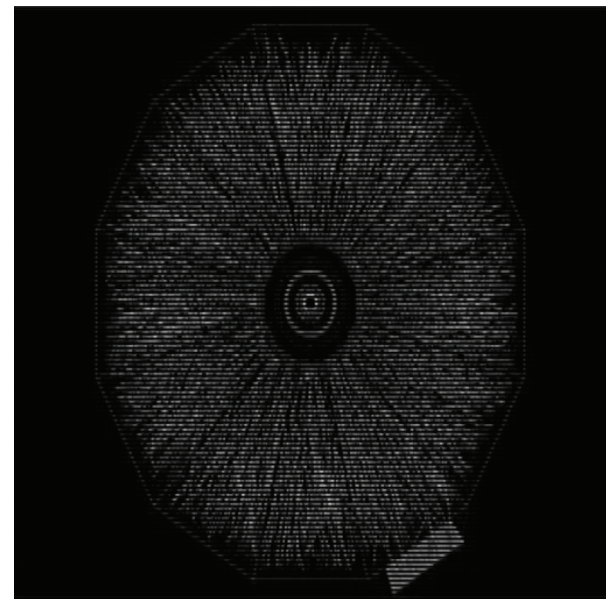

FIGURE 3: The reconstructed gray image of quark-gluon plasma. The data of the original image is encoded by the local frames of the fusion frame given by Example 14. The first coefficient of every local vector is deleted. The remained data is decoded by the dual fusion frame obtained by Example 14 .

\section{Conclusion}

We found that fusion frames can be used for experimental data transmission coding of high energy physics and studied the application of fusion frames in this field. For this goal, we first investigated the characteristics of fusion frames. We researched a class of alternate fusion duals of a given fusion frame and obtained some results about these duals. We provided a method for the matrix representation of the fusion frame operator of a given fusion frame system in a finitedimensional Hilbert space. Based on these results, we gave an algorithm for the construction of the dual fusion frame system with its local dual frames. A simulation example has been given to show the coding effect of a fusion frame system and its dual constructed by our methods when data erasure occurs in the transmission process.

\section{Acknowledgments}

The authors are thankful to the anonymous reviewers for their valuable comments and suggestions that have improved the presentation of this paper. This work was supported by the National Natural Science Foundation of China (11271001, 61170311), 973 Program (2013CB329404), and Sichuan Province Science and Technology Research Project (12ZC1802). be the analysis operator and synthesis operator of $F$, respectively; then

\section{References}

[1] S. Zeng, F. Z. Qi, and M. Wang, "Study and implementation of data transfer system in experiment of high energy physics," Computer Science, vol. 39, no. 6, pp. 93-95, 2012.

[2] E. J. Candés and D. L. Donoho, "New tight frames of curvelets and optimal representations of objects with piecewise $C^{2}$ singularities," Communications on Pure and Applied Mathematics, vol. 57, no. 2, pp. 219-266, 2004.

[3] B. G. Bodmann and V. I. Paulsen, "Frame paths and error bounds for sigma-delta quantization," Applied and Computational Harmonic Analysis, vol. 22, no. 2, pp. 176-197, 2007.

[4] C. H. Bennett, D. P. Divincenzo, and J. A. Smolin, "Capacities of quantum erasure channels," Physical Review Letters, vol. 78, no. 16, pp. 3217-3220, 1997.

[5] I. Cidon, H. Kodesh, and M. Sidi, "Erasure, capture, and random power level selection in multiple-access systems," IEEE Transactions on Communications, vol. 36, no. 3, pp. 263-271, 1988.

[6] A. F. Dana, R. Gowaikar, R. Palanki, B. Hassibi, and M. Effros, "Capacity of wireless erasure networks," IEEE Transactions on Information Theory, vol. 52, no. 3, pp. 789-804, 2006.

[7] B. G. Bodmann and V. I. Paulsen, "Frames, graphs and erasures," Linear Algebra and Its Applications, vol. 404, pp. 118-146, 2005.

[8] R. B. Holmes and V. I. Paulsen, "Optimal frames for erasures," Linear Algebra and Its Applications, vol. 377, pp. 31-51, 2004.

[9] J. Leng and D. Han, “Optimal dual frames for erasures II," Linear Algebra and Its Applications, vol. 435, no. 6, pp. 1464-1472, 2011.

[10] J. Leng, D. Han, and T. Huang, "Optimal dual frames for communication coding with probabilistic erasures," IEEE Transactions on Signal Processing, vol. 59, no. 11, pp. 5380-5389, 2011.

[11] J. Leng, D. Han, and T. Huang, "Probability modelled optimal frames for erasures," Linear Algebra and Its Applications, vol. 438, no. 11, pp. 4222-4236, 2013.

[12] T. Strohmer and R. W. Heath, Jr., "Grassmannian frames with applications to coding and communication," Applied and Computational Harmonic Analysis, vol. 14, no. 3, pp. 257-275, 2003.

[13] A. Albanese, J. Blömer, J. Edmonds, M. Luby, and M. Sudan, "Priority encoding transmission," IEEE Transactions on Information Theory, vol. 42, no. 6, pp. 1737-1744, 1996.

[14] B. G. Bodmann, "Optimal linear transmission by lossinsensitive packet encoding," Applied and Computational Harmonic Analysis, vol. 22, no. 3, pp. 274-285, 2007.

[15] P. G. Casazza and G. Kutyniok, "Frames of subspaces," in Wavelets, Frames and Operator Theory, vol. 345 of Contemporary Mathematics, pp. 87-113, American Mathematical Society, Providence, RI, USA, 2004. 
[16] P. G. Casazza, G. Kutyniok, and S. Li, "Fusion frames and distributed processing," Applied and Computational Harmonic Analysis, vol. 25, no. 1, pp. 114-132, 2008.

[17] P. G. Casazza and M. Fickus, "Minimizing fusion frame potential," Acta Applicandae Mathematicae, vol. 107, no. 1-3, pp. 7-24, 2009.

[18] P. G. Casazza, M. Fickus, D. G. Mixon, Y. Wang, and Z. Zhou, "Constructing tight fusion frames," Applied and Computational Harmonic Analysis, vol. 30, no. 2, pp. 175-187, 2011.

[19] P. G. Casazza and G. Kutyniok, "Robustness of fusion frames under erasures of subspaces and of local frame vectors," in Radon Transforms, Geometry, and Wavelets, vol. 464 of Contemporary Mathematics, pp. 149-160, American Mathematical Society, Providence, RI, USA, 2008.

[20] P. Găvruța, "On the duality of fusion frames," Journal of Mathematical Analysis and Applications, vol. 333, no. 2, pp. 871879, 2007.

[21] J. Leng and D. Han, "Orthogonal projection decomposition of matrices and construction of fusion frames," Advances in Computational Mathematics, vol. 38, no. 2, pp. 369-381, 2013.

[22] P. G. Massey, M. A. Ruiz, and D. Stojanoff, "The structure of minimizers of the frame potential on fusion frames," The Journal of Fourier Analysis and Applications, vol. 16, no. 4, pp. 514-543, 2010.

[23] P. G. Casazza, G. Kutyniok, S. Li, and C. J. Rozell, "Modeling sensor networks with fusion frames," in Wavelets XII, vol. 6701 of Proceedings of SPIE, San Diego, Calif, USA, August 2007.

[24] A. Chebira, M. Fickus, and D. G. Mixon, "Filter bank fusion frames," IEEE Transactions on Signal Processing, vol. 59, no. 3, pp. 953-963, 2011.

[25] B. G. Bodmann and G. Kutyniok, "Erasure-proof transmissions: fusion frames meet coding theory," in Wavelets XIII, vol. 7446 of Proceedings of the SPIE, San Diego, Calif, USA, August 2009.

[26] B. G. Bodmann, D. W. Kribs, and V. I. Paulsen, "Decoherenceinsensitive quantum communication by optimal $C^{*}$-encoding," IEEE Transactions on Information Theory, vol. 53, no. 12, pp. 4738-4749, 2007.

[27] R. Calderbank, P. G. Casazza, A. Heinecke, G. Kutyniok, and A. Pezeshki, "Sparse fusion frames: existence and construction," Advances in Computational Mathematics, vol. 35, no. 1, pp. 1-31, 2011.

[28] D. Han, K. Kornelson, D. Larson, and E. Weber, Frames for Undergraduates, vol. 40 of Student Mathematical Library, American Mathematical Society, Providence, RI, USA, 2007. 

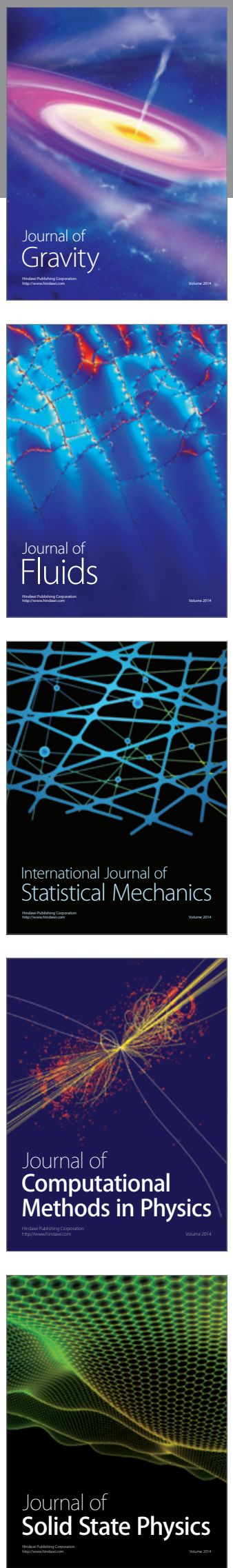

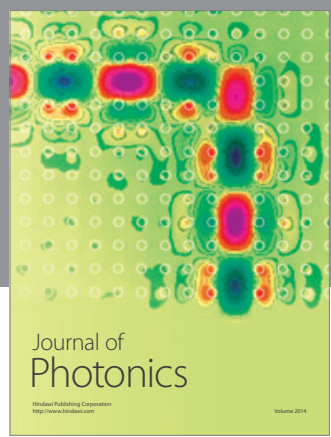

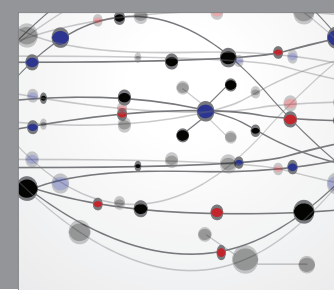

The Scientific World Journal

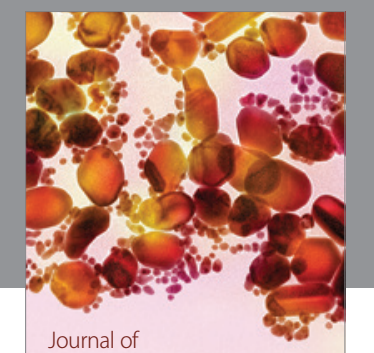

Soft Matter
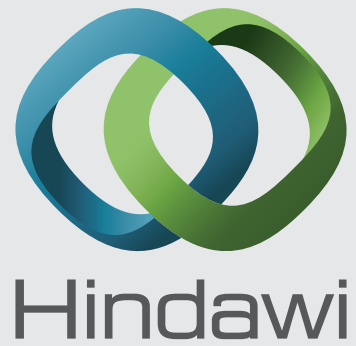

Submit your manuscripts at

http://www.hindawi.com
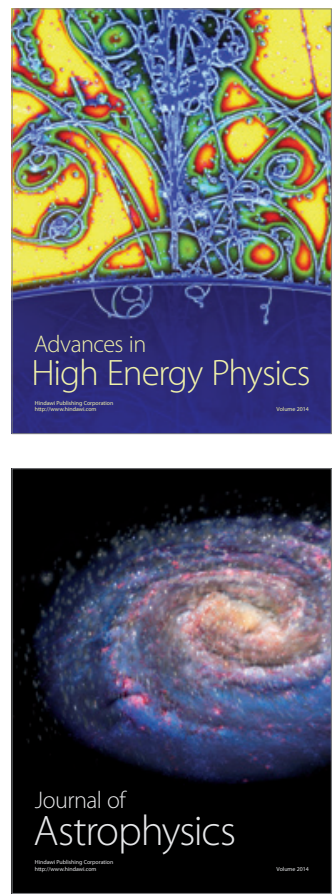
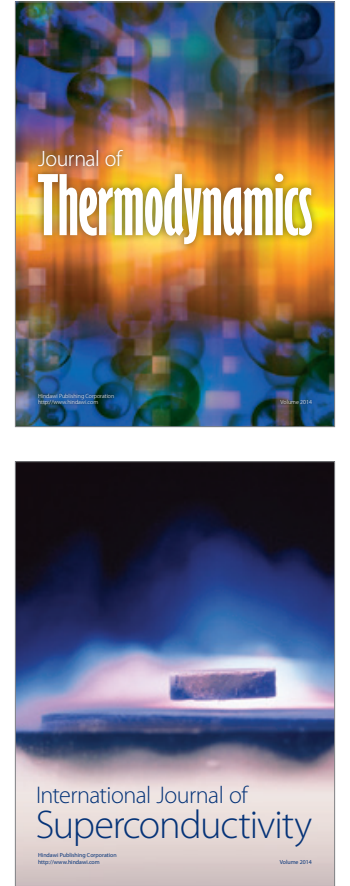
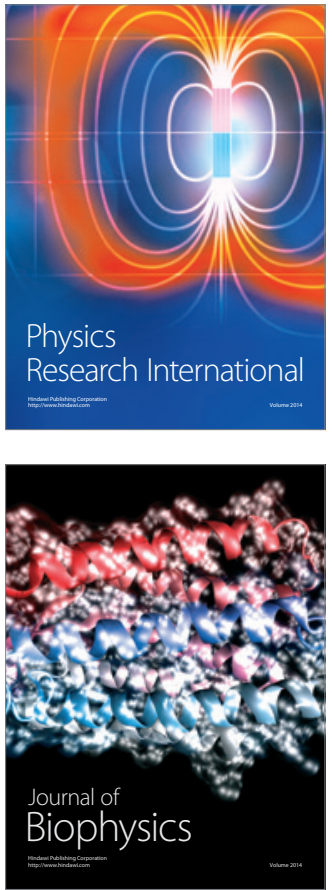
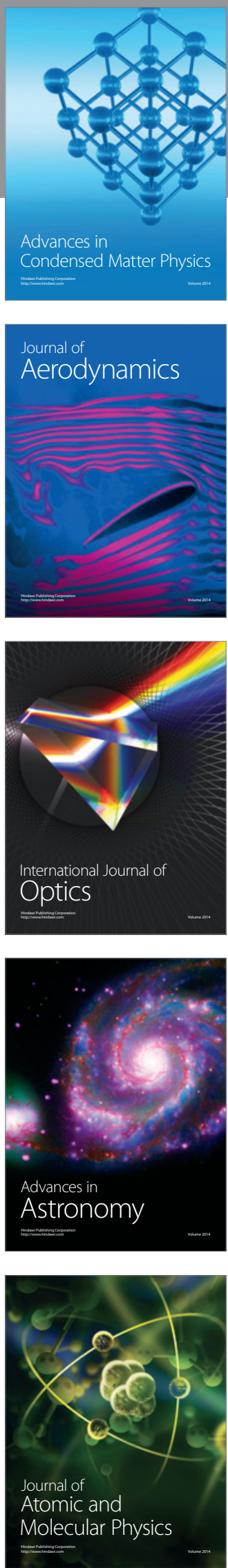